\section{Vorsicht mit „Ekzemen“ an der Brustwarze}

Eine 91-jährige Frau mit bekannter Familienanamnese für ein Mammakarzinom wurde wegen ihres seit zwöf Monaten bestehenden Erythems der linken Brust und der Brustwarze in eine senologische Einrichtung überwiesen. Die Gynäkologen mussten die Diagnose „Morbus Paget" stellen.

E in Versuch mit einer steroidhaltigen Salbe war bei der Patientin ohne Erfolg geblieben. Bei der Untersuchung zeigte sich eine rötliche, verkrustete Läsion mit partieller Zerstörung der Brustwarze. Die Hautbiopsie bestätigte den Verdacht auf ein lokales Carcinoma in situ und einen Morbus Paget der Brustwarze. Die Patienten unterzog sich einer linksseitigen Mastektomie.

Der Verdacht auf einen Morbus Paget (Pagetkarzinom) der Brustwarze ist gege- ben bei Patientinnen über 50, Einbeziehung des Warzenhofes und der Brustwarze mit Zerstörung der Warze und ausbleibender Besserung nach einem vierwöchigen Behandlungsversuch mit kortikoidhaltigen Lokalpräparaten. Unter diesen Umständen empfiehlt sich in jedem Fall die Überweisung an ein Brustzentrum.

Kommentar: Die lange Latenz ist vor allem bei Patientinnen im hohen Lebens-

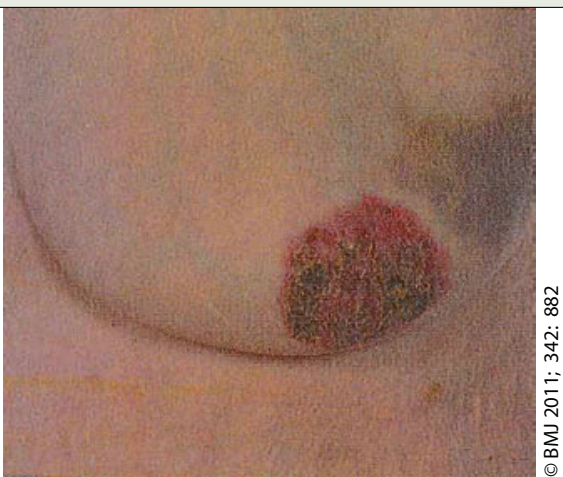

Das „Ekzem“ war ein Karzinom.

alter nicht ungewöhnlich. Viele betagte Frauen haben immer noch eine gewisse Scham, Brüste und Genitale einem Arzt zu präsentieren, ja sie vermeiden es sogar, sich selbst an diesen Körperstellen zu betrachten. Manche sind auch der Ansicht, Gynäkologen wären nur für jüngere Frauen da.

Prof. Dr. Hermann Füeßl

Ng ATW, Irvine T. BMJ 2011; 342: 882.

\title{
Antibiotika-Pflaster stoppt Lyme-Borreliose
}

Die Prophylaxe mit Antibiotika kann eine Lyme-Borreliose vermeiden helfen. Doch wer nimmt nach einem Zeckenbiss drei bis vier Wochen lang Antibiotika ein? Jetzt könnte eine systemische Behandlung überflüssig werden: Die lokale Therapie mit Azithromycin scheint für die sichere Eradikation der Erreger in der Haut auszureichen.

st die lokale Antibiotika-Therapie die Strategie der Zukunft für die LymeBorreliose-Prophylaxe? Im Tierversuch zumindest gelang es Münchner Wissenschaftlern von der Ludwig-MaximiliansUniversität, den Erreger in der Haut mit einer lokalen Antibiotikatherapie sicher zu eliminieren.

Der Bakteriologe Reinhard Straubinger und sein Team infizierten 78 Mäuse mit dem Erreger Borrelia burgdorferi. Sie inokulierten entweder den Erreger mit einer Nadel oder ließen infizierte Zecken die Versuchstiere beißen. Anschließend

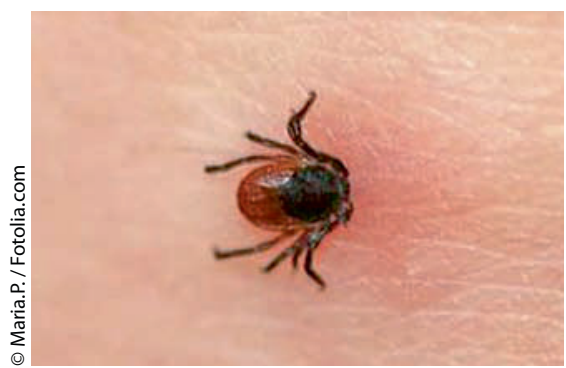

Borreliose: Lokale Therapie ausreichend? klebten sie den Mäusen ein mit Azithromycin-Gel beschichtetes Pflaster oder Placebo auf die Stelle des Nadel- oder Zeckenstichs. Von den 40 Mäusen, die über einen Nadelstich infiziert wurden, erhielten je zehn Tiere ein Pflaster mit einer 5\%igen Azithromycin-Formulierung eine Stunde, drei Tage oder fünf Tage lang nach Infektion aufgeklebt. Die anderen Mäuse wurden mit Placebo behandelt.

Von den Tieren, die direkt von Zecken gebissen worden waren, erhielten neun über das Pflaster eine 4\%ige Antibiotikaformulierung, acht Tiere ein 10\%iges und fünf Mäuse ein 20\%iges Gel. Der Rest wurde entweder gar nicht behandelt oder bekam ein Placebo aufgeklebt. Mit der Behandlung wurde direkt nach Entfernen der Zecke begonnen.

Die lokale Therapie überzeugte: Bei keinem Tier aus der Gruppe mit Zeckenbiss ließen sich im Anschluss an die Behandlung noch Erreger nachweisen. Gleiches galt für die Mäuse, die über einen Nadelstich infiziert worden waren - vo- rausgesetzt, die Behandlung erfolgte innerhalb von drei Tagen nach der Infektion. Die gute Wirksamkeit der topischen Behandlung auch bei verzögertem Beginn erklären die Studienautoren zum einen mit der Art der Erregervermehrung in der Haut: Es ist bekannt, dass die Spirochäten zunächst einmal zwei Tage und länger am Infektionsort verbleiben, ehe sie sich im gesamten Organismus ausbreiten. Zum anderen konnten die Forscher zeigen, dass die Gewebekonzentration von Azithromycin nach Applikation mehr als 3.800fach höher war als die minimale Hemmkonzentration der Bakterien.

Fazit: Mittlerweile wird die lokale Antibiotika-Behandlung gegen Borrelien auch mit infizierten Menschen in einer PhaseIII-Studie getestet. Jetzt bleibt zu hoffen, dass diese Untersuchung die gute Wirksamkeit und geringe Nebenwirkungsrate bestätigt - die wichtigste Voraussetzung für den präventiven Einsatz von Medikamenten.

Dr. Dagmar Kraus

Knauer J et al. Evaluation of the preventive capacities of a topically applied azithromycin formulation against Lyme Borreliosis in a murine model. J Antimicrob Chemother 2011, Sept 15 [Epub ahead of print] 